International Journal of Applied Dental Sciences 2021; 7(3): 387-392

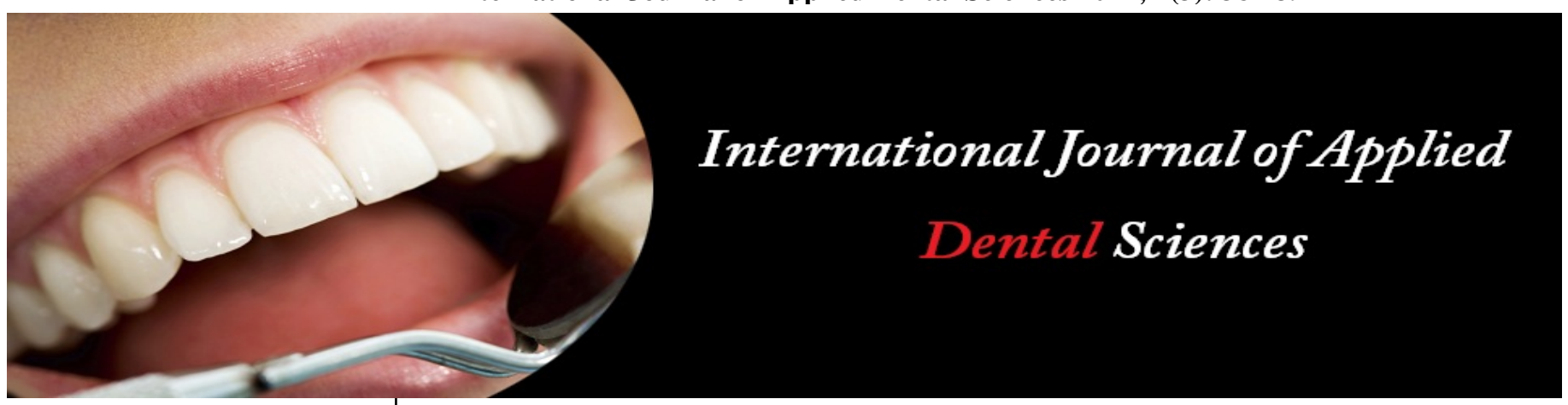

ISSN Print: 2394-7489

ISSN Online: 2394-7497

IJADS 2021; 7(3): 387-392

(C) 2021 IJADS

www.oraljournal.com

Received: 14-05-2021

Accepted: 16-06-2021

Shen Furtado

Postgraduate student,

Department of Conservative

Dentistry and Endodontics,

Y.M.T Dental College,

Maharashtra University of

Health Sciences, Navi Mumbai,

Maharashtra, India

Vibha Hegde

Professor and Head, PG Guide,

Department of Conservative

Dentistry and Endodontics,

Y.M.T Dental College,

Maharashtra University of

Health Sciences, Navi Mumbai,

Maharashtra, India

Ushaina Fanibunda

Reader and PG Guide,

Department of Conservative

Dentistry and Endodontics,

Y.M.T Dental College,

Maharashtra University of

Health Sciences, Navi Mumbai,

Maharashtra, India
Corresponding Author:

Shen Furtado

Postgraduate student,

Department of Conservative

Dentistry and Endodontics,

Y.M.T Dental College,

Maharashtra University of

Health Sciences, Navi Mumbai,

Maharashtra, India

\section{Deep margin elevation: Raising the bar in adhesive restorations - A case series}

\author{
Shen Furtado, Vibha Hegde and Ushaina Fanibunda
}

DOI: https://doi.org/10.22271/oral.2021.v7.i3f.1327

\section{Abstract}

Treating subgingival deep carious defects can be clinically challenging owing to the difficulty in obtaining sufficient restorative access and a moisture-free field for bonding. Traditional restorative and surgical approaches are aggressive. In keeping with modern restorative materials and techniques, the deep margin elevation procedure has evolved as a simple and convenient means to manage hitherto difficult cases. Clinical cases detailing the step wise procedure for deep margin elevation have been described with emphasis on magnification and isolation as the key to success.

Keywords: Deep margin elevation, bulk fill composites, snow plow technique, preheated composite

\section{Introduction}

Advances in the material science, adhesive systems and improved isolation techniques have greatly expanded the clinical use and applications of composite restorative materials. With the help of minimally invasive techniques, even extensive and undermining defects can be restored, thereby preserving the sound tooth structure and providing long-lasting durability to the tooth. Furthermore, direct composite restorations frequently serve as the foundation upon which margins of indirect restorations are placed.

As clinicians, one of the most common yet challenging experiences faced in daily practice are, achieving durable restorations in the moist deep subgingival areas of Class 2 and Class 5 cavities. The challenges include difficulty in obtaining a well isolated operatory field, weaker bond strengths in cervical areas, difficulty in recording a proper impression and providing good contact and contours especially in restorations beyond the cemento-enamel-junction. If any of these steps are not properly executed, it may hamper the longevity of the restoration at the tooth-restorative interface leading to an eventual restorative failure ${ }^{[1]}$. However, recently it has been shown that if moisture control is possible, these problems can also be overcome using newer materials alongside modified techniques even in deeper cavities ${ }^{[2]}$.

The approaches to such clinical challenges are many. One such procedure is the crown lengthening procedure where the gingival margin can be surgically exposed by an apical displacement of the tissues ${ }^{[3]}$. However, the above procedure is associated with problems like violating the biologic width, loss of attachment and anatomical complications like a close proximity to root concavities and furcations. Once exposed to the oral environment, the gingival margins are challenging to maintain.

Another approach, though not used routinely, is orthodontic extrusion to coronally reposition and make accessible the sub-gingival lesions or defects. Orthodontic extrusion requires careful consideration of the crown root ratio and is time consuming. This approach may be better suited for anterior traumatized teeth with horizontal fractures ${ }^{[4]}$.

Another approach was presented by Dietschi and Spreafico, in 1998, who suggested that instead of surgically lengthening the crown or extruding the tooth which can be both time consuming as well as invasive, rather elevate the existing proximal margin, from a subgingival cavity to a more supragingival level by the use of an adequate layer of composite along with an appropriate isolation method. This was previously known as the open sandwich technique which has now morphed into a technique known as "cervical margin relocation" (CMR) ${ }^{[5]}$. This procedure is done under rubber dam isolation following the placement of an appropriate matrix and also reduces the difficulty of subsequent impression techniques and cementation procedures for indirect restorations. 
In 2012, Magne and Spreafico referred to this technique as "deep margin elevation" (DME) ${ }^{[1]}$. This was a landmark paper, which detailed the procedure in light of new materials and isolation strategies. Other terms such as "coronal margin relocation" and "proximal box elevation" can also be found in the literature.

The layer of these resin composites used to elevate the gingival margin in deep cavities may either be incorporated into the direct restoration or may serve as a base, over which an indirect composite or ceramic restorations can be fabricated [5]. Ghezzi et al., describe the deep margin elevation technique, as a more minimally invasive procedure that is both biologically sound and operatively friendly ${ }^{[6]}$.

The aim of this article is to present the results of three clinical cases to describe this new conservative protocol for DME that reconsiders the dogma of the biologic width.

\section{Materials and Method}

2.1 Case 1: A 14year old female patient was referred to the Department of Conservative Dentistry and Endodontics for the chief complaint of discoloration with respect to the right maxillary lateral incisor. Clinical examination revealed a defective previous restoration on the mesio-proximal aspect with an uneven surface texture of the restoration (Fig. 1 a). The restoration extended sub gingivally and there was a proximal overlap of the central and lateral incisor, making rerestoration challenging. Orthodontic treatment was in progress and had been temporarily suspended till restorative work was completed. The tooth was vital and non-tender to percussion. Radiographically, normal periapical architecture was present (Fig. 1 b). A decision to replace the restoration was made. The previous restoration was removed and its subgingival extent evaluated (Fig. $1 \mathrm{c}$ ). It was determined to be $1 \mathrm{~mm}$ below the marginal gingiva and suitable for DME. Gingivectomy was carried out using Gingiburs (Smart ROTO, Germany) and further hemostasis was obtained with Viscostat haemostatic agent (Fig. 1 d, e, f). The tooth was anesthetised and the anterior segment was isolated under the rubber dam. Additional retraction to expose the sub gingival margin of 12 was obtained with a B4 tissue retractor Brinker clamp (Fig. 1 g). A glass ionomer liner (Ionoseal, Voco $\mathrm{GmbH}$ ) was used to provide a therapeutic effect to the pulp (Fig. $1 \mathrm{~h}$ ). The cavity was then etched using 35\% phosphoric acid (Prime dental, India) and a universal bonding agent (Single bond universal adhesive, 3M, ESPE, USA) was applied (Fig. 1 i). A Bioclear anterior pre contoured plastic matrix (Bioclear matrix systems, South Warner St Tacoma, WA) was used to achieve proper contour (Fig. 1 j). Wedge placement was not required owing to the proximal overlap and since orthodontic intervention would change the subsequent position of the teeth. Injection moulding was done using a flowable resin and nanofilled composite material (3M Filtek Z350 XT Universal Restorative). The material was pre heated at $50{ }^{\circ} \mathrm{C}$ using a composite heating device (AR Heater Magic box, China) (Fig. $1 \mathrm{k}, \mathrm{l}, \mathrm{m})$. The restoration was finished and polished and the patient was recalled at 1 week, 3 months and 6 months. The gingiva was healthy, the restoration was intact and the orthodontic treatment was in progress (Fig. 1 n, o, p).

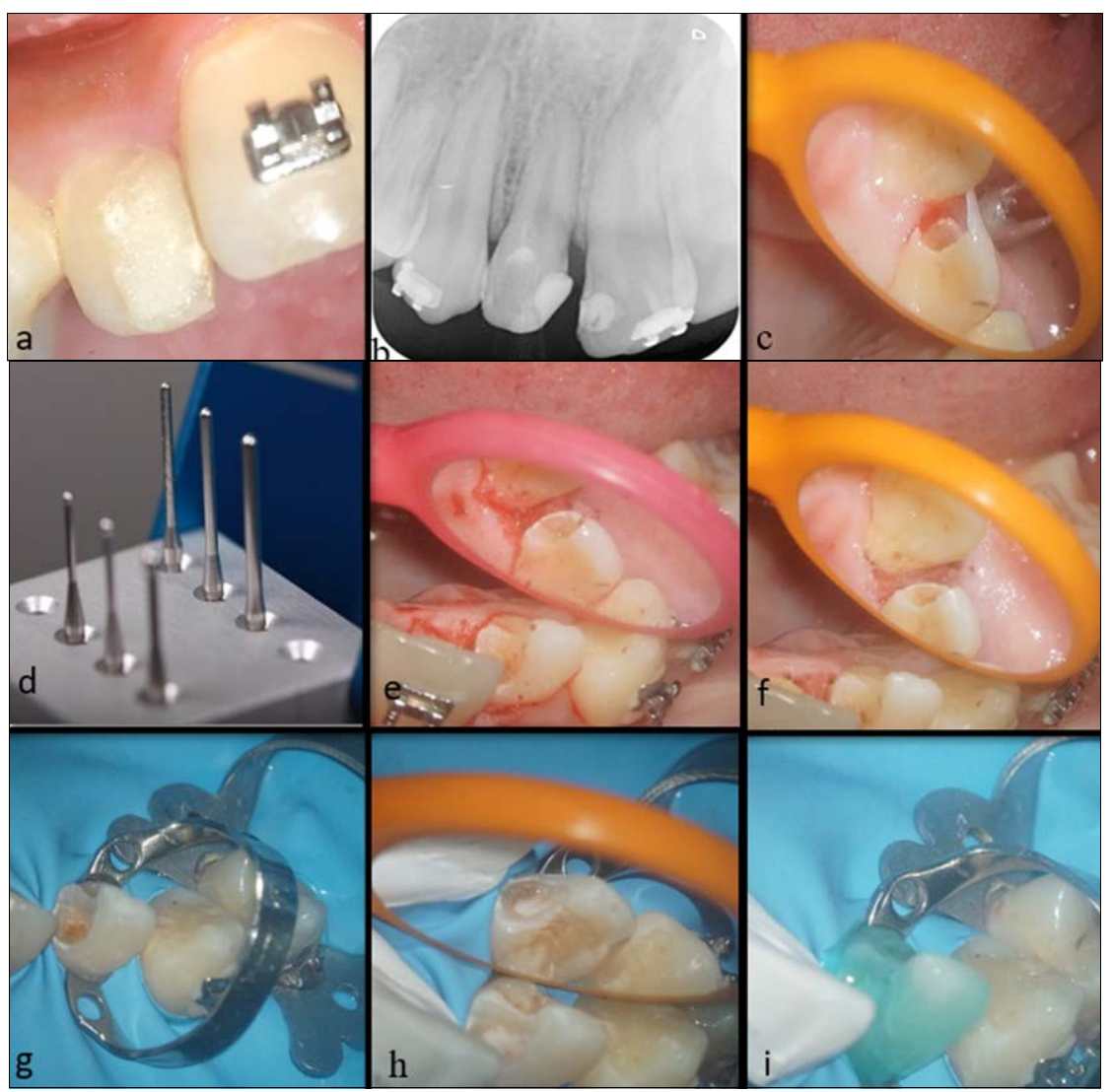

Fig 1: a) Preoperative clinical image showing presence of a defective restoration with 12. b) Radiographic image showing defective restoration with 12. c) Removal of faulty restoration \& subgingival margins visible. d) Gingiburs (Smart ROTO, Frank Dental, Germany) used for gingivectomy. Image source: https://smartroto.pl. e \& f) Gingivectomy performed using Gingiburs with good haemostatis. g) Isolation with additional B4 retractor clamp on 12. h) Application of Glass ionomer cement liner (Ionoseal,Voco GmbH). i) Application of etchant (Prime Dental, India). 

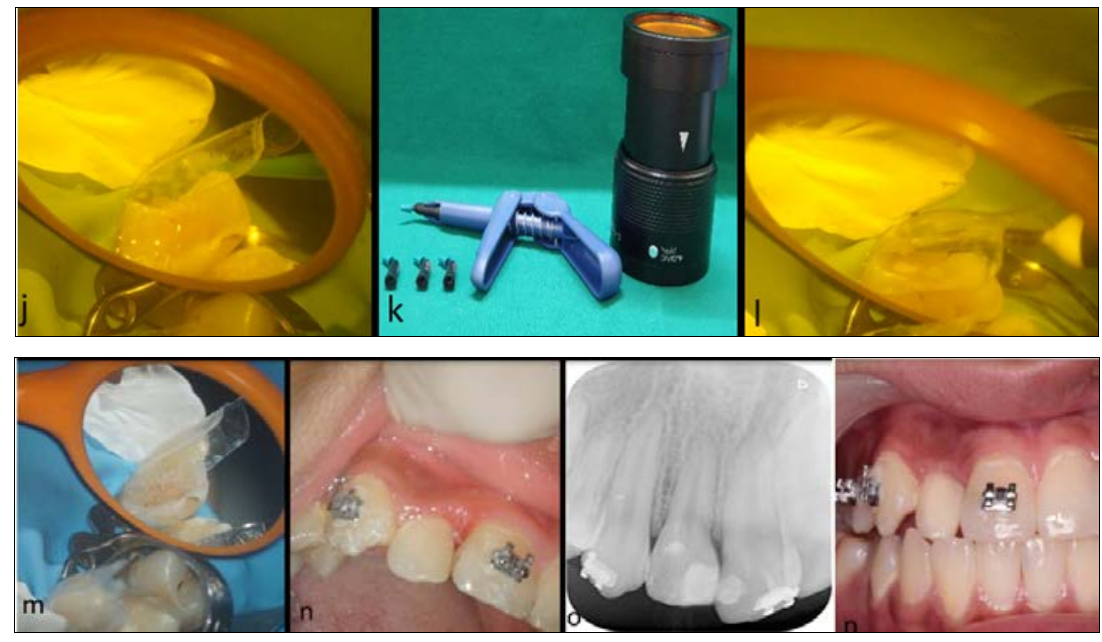

Fig 1: j) Application of Bioclear matrix with universal bonding agent. k) Preheating device used in injecting moulding technique. l)Application of flowable composite resin. m) Injection molding technique with preheating composite. $n$ ) Immediate post- operative clinical view after finishing and polishing. o) Immediate post- operative radiographic view. p) 6 months follow up

2.2. Case 2: A 25year old female patient reported to the Department of Conservative Dentistry and Endodontics with the chief complaint of pain with respect to the left maxillary second premolar since 2 weeks. Clinical examination revealed mesio-proximal caries with 25 (Fig. 2 a) and radiographic examination confirmed deep mesio-proximal caries involvement approaching the pulp chamber and root canal treatment was planned (Fig. 2 b). The first premolar (24) was previously endodontically treated which was underfilled due to which the patient was advised a retreatment with 24 . No other periapical pathology was recorded. Post the administration of a short acting anesthetic (2\% lidocaine), caries was excavated completely, exposing subgingival margins and gingivectomy was performed using Gingiburs as used in the previous case (Fig. 2 c). The cavity could now be isolated under the rubber dam (Fig. 2 d) and teflon tape was used to further isolate the gingival margin. A sectional matrix system (Palodent) was used. Owing to inadequate space at the deep cervical margin to place a wedge, Teflon floss was used to adapt the band at the gingival margin (Fig. 2 e). The cavity was then etched using 35\% phosphoric acid and bonded using a universal bonding agent (Single bond universal adhesive, 3M, ESPE, USA). In this case, the margins were elevated using Snowplow Technique (i.e. incorporation of flowable along with packable composite restorations (Filtelk bulk fill flow able with Filtek Z330XT Universal restorative, 3M, ESPE, USA) as a gingival liner followed by pre-endodontic build up (Fig. 2 f, g, h). Conventional endodontic therapy was carried out, post endodontic restoration with Z350XT was done and an Emax crown was delivered with the same (Fig. 2 $\mathrm{i}, \mathrm{j}, \mathrm{k}, \mathrm{l}$ ). The patient was kept on follow up. Gingival health was recorded at subsequent appointments and found to be satisfactory.

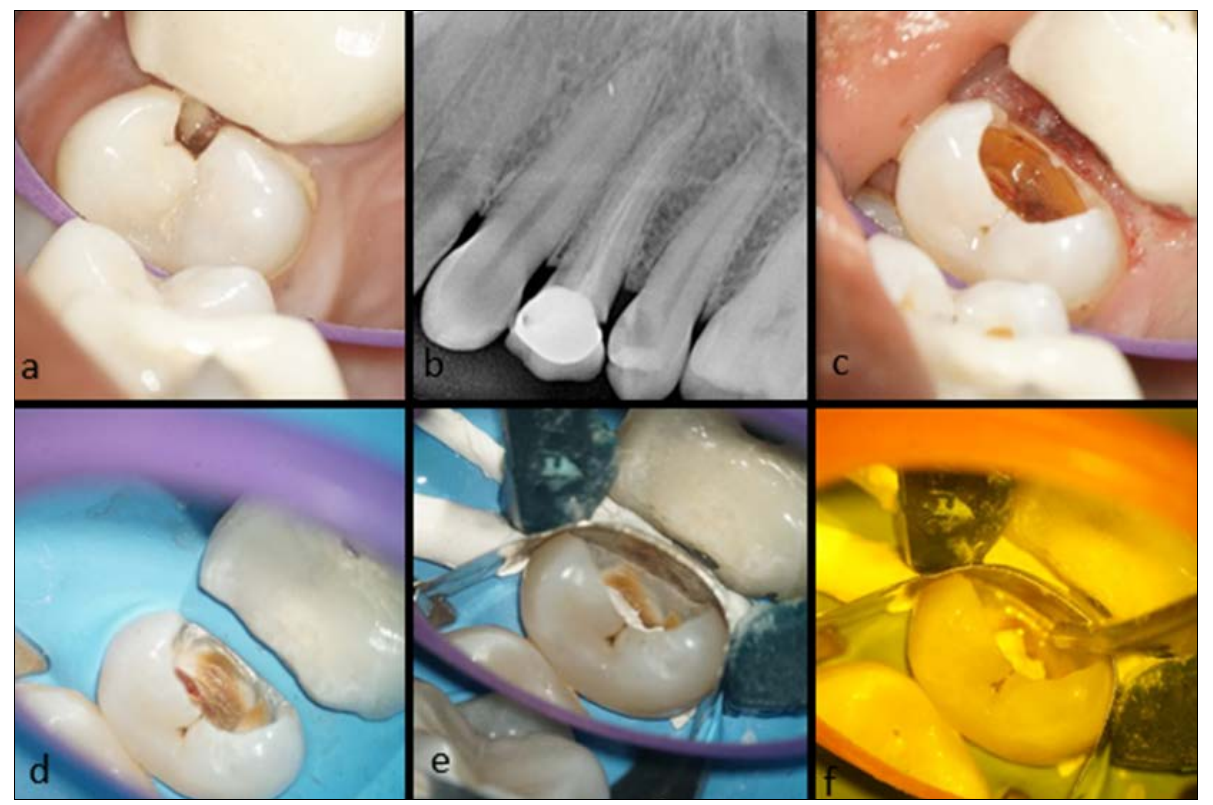

Fig 2: a) Pre-operative clinical view with a mesial subgingival margin. b) Radiographic evaluation of the extent of caries involvement with 25. c) Excavation of caries and gingivectomy done using gingiburs. d) Rubber dam isolation done. e) Application of saddle matrix using Teflon floss and Palodent ring. f) Application of flowable composite for DME in snowplow technique. 


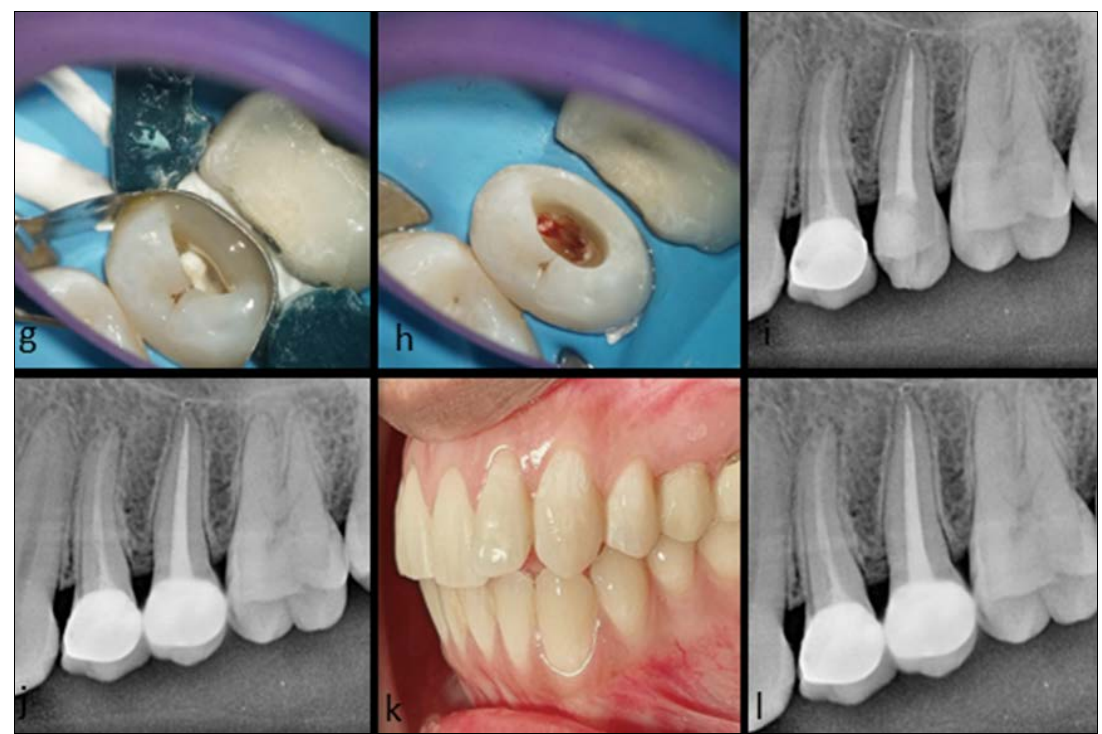

Fig 2: g) Application of packable composite for DME in snowplow technique. h) Completion of pre-endodontic buildup \& initiation of root canal treatment. i) Radiograph showing DME with preendo build up \& completed root canal therapy with 25. j) Post-operative radiograph. k) Emax full crowns delivered with 25. l) Radiographic image showing 6 months follow up.

2.3. Case 3: A 37year old male patient reported to the Department of Conservative Dentistry and Endodontics with the chief complain of a fractured restoration, food lodgement and a mild sensitivity with respect to the right mandibular first molar. Clinical examination revealed a fractured amalgam restoration with respect to the disto-proximal surface with marginal gaps at the tooth-restorative interface (Fig. 3 a). Radiographic evaluation revealed the presence of secondary caries below the gingival seat and a close proximity of the restoration to the distal pulp horn. The lamina dura and the periapical status were unremarkable (Fig. 3 b). Pulp sensibility test confirmed the status of the pulp to be vital. Post the administration of a short acting anesthetic (2\% lidocaine), the cavity was then well isolated using a rubber dam (Fig. 3 c). Post removal of the past restoration, undermined infected dentin (secondary caries) was present below the gingival seat (Fig. $3 \mathrm{~d}$ ) which on removal resulted in a subgingival cavity margin (Fig. 3 e). The margin was then elevated with a sectional matrix system (Palodent) along with the use of bulk fill composites (BFCs) in snowplow technique (Filtelk bulk fill flowable with Filtek Z330XT Universal restorative, 3M, ESPE, USA)and brought to a supragingival level (Fig. $3 \mathrm{f}$ ). This level was then verified radiographically for a good adaptation over which an indirect ceramic restoration was fabricated (Fig. $3 \mathrm{~h}, \mathrm{i}, \mathrm{j}$ ). The last image shows a 6months follow up of the restoration without any initiation of secondary decay and a sound periapical status too (Fig. $3 \mathrm{k}$ ).

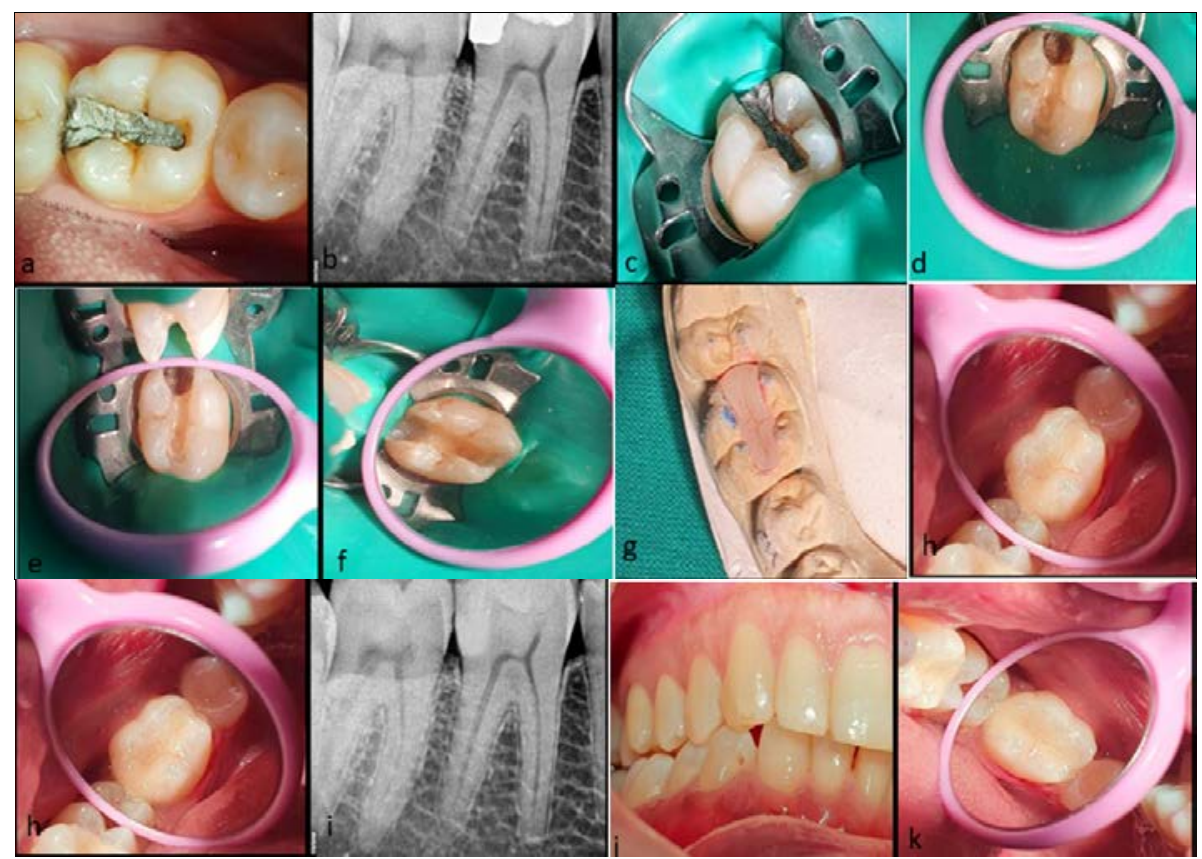

Fig 3: a) Clinical image showing the fractured amalgam restoration. b) Pre-operative radiographic image showing proximity to the distal pulp horn. c) Rubber dam isolation done. d) Presence of undermined caries below the past restoration. e) Post caries excavation. f) Post deep margin elevation using bulk fill composites in snowplow technique. g) Prefabricated indirect restoration on the diagnostic cast. h) Post cementation of the indirect restoration. i) Radiographic appearance showing the indirect restoration over an elevated margin. j) Evaluation of the bite post cementation. k) 6 months follow up post cementation. 


\section{Discussion}

The management of subgingival lesions or defects invading the biologic width pose a clinical challenge and are managed by surgical crown lengthening or orthodontic extrusion procedures. Recent advances in adhesive technology, material science and isolation techniques have made possible the conservative deep margin elevation approach. Three case reports are presented which describe the clinical step-wise procedure for the successful and expeditious management of these cases by the DME approach.

The DME approach is suitable for a wide range of clinical situations, where the subgingival defects are limited to the junctional epithelium and has not invaded the connective tissue attachment i.e., the gingival margin of the future restoration is estimated clinically to be at least at approximately $1-1.5 \mathrm{~mm}$ distance from the alveolar crest. The biologic width is the height between the deepest area or point of the gingival sulcus and the alveolar bone crest. The mean biologic width is $2.04 \mathrm{~mm}$, of which $1.07 \mathrm{~mm}$ makes the connective tissue attachment and another approximate 0.97 $\mathrm{mm}$ is occupied by the junctional epithelium ${ }^{[7]}$. Historically it has been believed that any violation to this area would cause a deleterious effect to the supporting periodontium ${ }^{[8]}$. However, this dogma of the biologic width has been revisited and today it is largely held that a smooth hard well-sealed restorative surface is not deleterious adjacent to the gingival attachment apparatus provided it is limited to the junctional epithelium and does not invade the connective tissue attachment. Invasion of the connective tissue component results in gingival inflammation and recession ${ }^{[9]}$. Moreover, Schmidt et al. and Maynard and Wilson (1979) have shown that the dimensions of the biologic width are not fixed numbers but rather a variable clinical entity with considerable inter and intra individual variation ${ }^{[10,11]}$.

As stated by Ghezzi et al. the ability to clinically isolate the subgingival lesion under a rubber dam should guide the choice of treatment procedure rather than the rigid concept of geometric biology. ${ }^{6}$ The first and second case presented in this paper fall into the Type IIa category of DME cases as classified by Ghezzi et al. Type IIa refers to cases limited in depth to the epithelial component and isolatable clinically by gingivectomy or raising a flap. The third case is of Type I category i.e., deep cases isolatable without gingival soft tissue management. Type III refers to cases involving the connective tissue component necessitating the classic crown lengthening osteotomy approach.

In the first and second case, the subgingival carious lesion was clinically isolatable after gingivectomy to expose the sub gingival cavity margin. Based on the distance between the cavity margin and bone crest, evaluated radiographically and confirmed clinically in both cases it was determined that the connective tissue component of the biologic width was not invaded. In the third case the subgingival margin could be exposed without the need for gingivectomy

Gingivectomy may be carried out either with a scalpel blade, special soft tissue trimming burs, a laser or electrocautery. Tissue trimming burs maybe either zirconia or smooth stainless-steel burs. We used Gingiburs (Smart ROTO, Frank. Dental, Germany) which are smooth stainless-steel burs intended to trim the gingival soft tissue and the heat produced enables coagulation and limited bleeding. These burs are convenient, inexpensive and safe to use.

Other procedural aspects incorporated in our cases included magnification, isolation and appropriate matrix band adaptation. Magnification either in the form of loupes (2.5- 3.5x) or a dental operating microscope at mid-range magnification is of utmost importance for the successful outcome of the procedure as it enables precise visualization of carious extent, conservation of tooth structure, visualization of matrix adaptation, moisture leakage if any and aids in achieving a smooth restorative interface. Rubber dam isolation was done using a heavy rubber dam sheet (Nictone) for good tissue retraction and the additional use of tissue retractor clamps (as seen in Case 1) and floss ties may be considered depending on the case. Complete isolation not only from saliva but also from blood and gingival crevicular fluid must be ensured and Teflon placement at the gingival margin (as seen in Case 2) is a valuable adjunct in this regard. Precontoured matrix bands are of benefit in this procedure either the plastic for example, Bioclear matrices or the saddle matrix for example, from $\mathrm{T}$ or VM or the sectional matrices for example from Palodent or Garrison are preferred to the conventional Tofflemire or Ivory matrix bands. The wedge maybe either the wooden wedge which can be customized and absorbs moisture too, or the recently introduced plastic wedges of sectional matrix systems for example, Bioclear, Palodent or Garrison systems which can adapt to the tooth surface to some extent. The Bioclear system also has a special diamond wedge for deep restorations. The banana matrix band which is akin to a modified tofflemire band, can be used as it provides tight adaptation at the neck of the tooth ${ }^{[1]}$. It can be followed by the use of a precontoured band for obtaining a good contact in direct restorative cases. In very deep cases, where there is inadequate space just below the cervical margin for a wedge, the use of teflon floss (as seen in Case 2) is an excellent adjunct to adapt the band to the tooth [12].

A universal bonding agent was employed in our cases. The universal bonding agent (Single bond universal adhesive, 3M, ESPE, USA), with the chemical advantage of 10-MDP provides a durable dentinal bond with less moisture sensitivity and maybe beneficial for bonding to the heterogenous dentine-cementum tissues in the moist subgingival milieu ${ }^{[13]}$. The use of viscoelastic flowable composite liners has been advocated at the gingival margin of proximal cavities to overcome the polymerization shrinkage stresses which accumulate at this critical interface. In both cases a bulk fill flowable liner (3M Filtek Bulk fill Flowable) was preferred owing to its low polymerization shrinkage and a nanofilled restorative composite 3M Z350XT was used incrementally for its superior strength and polish ability. Other materials that that can also be used for DME include silver amalgam since they are less technique sensitive as well as resin modified glass iononer cement (RMGIC). But RMGIC lacks sufficient strength to withstand masticatory forces and have inferior mechanical properties as compared to contemporary composite resin materials. In the first case, the bulk fill composites were preheated to reduce their viscosity for better marginal adaptation into the line and point angles without compromising on its mechanical properties ${ }^{[14]}$. Recently the snow plow technique which utilises a combination of a flowable liner and packable composite has been introduced to obtain the dual benefit of the viscoelastic flowable composite liner which adapts well and the packable composite with superior mechanical properties. We employed the snow plow technique in the second case ${ }^{[15]}$.

In indirect restorations, the DME approach elevates the gingival margin and makes it possible to record the elevated margin by impressions (as seem in Case 3) or intra oral scanning, A direct composite base minimizes the sacrifice of 
sound tooth structure by filling the undercuts, provides a good geometry to the inlay/onlay restorations and supports the undermined cusps ${ }^{[16]}$.

In the cases presented, even though the margins were deep, there was no evidence of gingival inflammation or plaque accumulation at subsequent follow up appointments. Utmost care was taken to ensure smooth polished margins at the tooth restorative interface. The patients were counselled regarding the maintenance of meticulous oral hygiene. Brunsvold and Lane showed that in the absence of marginal overhangs, no association could be drawn between subgingival margins and secondary caries ${ }^{[17]}$. According to Frese et al., even though drawing conclusions about an epithelial reattachment over a polished composite surface is not definite, but composites being biocompatible, a non-inflammatory rehabilitation of the tooth with respect to the periodontium can definitely be achieved ${ }^{[18]}$. Our case reports have shown that the violation of the biological width to a limited extent, can be clinically successful provided oral hygiene is well maintained and a smooth polished restorative interface is provided.

In both direct and indirect restorative procedures, the lack of ferrule on the surface of the tooth requiring deep margin elevation may preclude the wide usage of this procedure on the buccal and lingual tooth surfaces for structural integrity concerns. Nevertheless, it provides an expeditious and convenient clinical technique eliminating the need for surgical, invasive or other long term procedures. Further clinical studies to determine the long term performance of this treatment approach should be encouraged.

\section{Conclusion}

Deep margin elevation is a conservative and reliable technique to manage sub-gingival defects, when performed with meticulous attention to detail to ensure a smooth hard well-polished and sealed restorative surface against which a healthy periodontium can adapt and survive. It is a relatively non-invasive and a less time-consuming approach and should be considered when applicable.

\section{References}

1. Magne P, Spreafico RC. Deep margin elevation: A paradigm shift. Am J Esthet Dent 2012;2(2):86-96.

2. Kuper NK, Opdam NJ, Bronkhorst EM, Huysmans MC. The infsluence of approximal restoration extension on the development of secondary caries Journal of Dentistry 2012;40(3):241-247.

3. Hempton TJ, Dominici JT. Contemporary crownlengthening therapy: a review. The Journal of the American Dental Association 2010;141(6):647-55.

4. Koyuturk AE, Malkoc S. Orthodontic extrusion of subgingivally fractured incisor before restoration. A case report: 3-years follow-up. Dental traumatology 2005;21(3):174-8.

5. Dietschi D, Spreafico R. Current clinical concepts for adhesive cementation of tooth-colored posterior restorations. Practical Periodontics and Aesthetic Dentistry 1998;10:47-54.

6. Ghezzi C, Brambilla G, Conti A, Dosoli R, Ceroni F, Ferrantino L. Cervical margin relocation: case series and new classification system. The international journal of esthetic dentistry 2019;14(3):272-84.

7. Jepsen S, Caton JG, Albandar JM, Bissada NF, Bouchard $\mathrm{P}$, Cortellini $\mathrm{P}$ et al. Periodontal manifestations of systemic diseases and developmental and acquired conditions: Consensus report of workgroup 3 of the 2017
World Workshop on the Classification of Periodontal and Peri-Implant Diseases and Conditions. Journal of clinical periodontology 2018;45:S219-29.

8. Nevins M, Skurow HM. The intracrevicular restorative margin, the biologic width, and the maintenance of the gingival margin. Int J Periodont Restor Dent 1984;4:3049.

9. Jorgic-Srdjak K, Plancak D, Maricevic T, Dragoo MR, Bosnjak A. Periodontal and prosthetic aspect of biological width part I: Violation of biologic width. Acta Stomatol Croat 2000;34:195-7.

10. Schmidt JC, Sahrmann P, Weiger R, Schmidlin PR, Walter C. Biologic width dimensions-a systematic review. Journal of clinical periodontology 2013;40(5):493-504.

11. Maynard Jr JG, Wilson RD. Physiologic dimensions of the periodontium significant to the restorative dentist Journal of Periodontology 1979;50(4):170-174.

12. https://contact-points-ambassador.teachable.com/ \& https://youtu.be/u16rST2H5sk. accessed: (31.08.21)

13. Hattan MA, Pani SC, Al Omari M. Composite bonding to stainless steel crowns using a new universal bonding and single-bottle systems. International journal of dentistry 2013.

14. Deb S, Di Silvio L, Mackler HE, Millar BJ. Pre-warming of dental composites. Dental materials 2011;27(4):e51-9.

15. Doustfateme S, Khosravi K, Hosseini S. Comparative evaluation of microleakage of bulk-fill and posterior composite resins using the incremental technique and a liner in Cl II restorations. Journal of Islamic Dental Association of Iran 2018;30(1):1-8.

16. Moscovich H, Roeters FJ, Verdonschot N, De Kanter RJ, Creugers NH. Effect of composite basing on the resistance to bulk fracture of industrial porcelain inlays. Journal of dentistry 1998;26(2):183-9.

17. Brunsvold MA, Lane JJ. The prevalence of overhanging dental restorations and their relationship to periodontal disease. Journal of clinical periodontology 1990;17(2):67-72.

18. Frese C, Wolff D, Staehle HJ. Proximal box elevation with resin composite and the dogma of biological width: clinical R2-technique and critical review. Operative Dentistry 2014;39(1):22-31. 